\title{
Neutrophil-targeted, protease-activated pulmonary drug delivery blocks airway and systemic inflammation
}

\author{
Joscelyn C. Mejías, ${ }^{1,2,3}$ Osric A. Forrest, ${ }^{4,5}$ Camilla Margaroli, ${ }^{4,5}$ David A. Frey Rubio, ${ }^{1,2,3}$ Liliana Viera, $^{6}$ \\ Jindong Li, ${ }^{6}$ Xin Xu, ${ }^{6}$ Amit Gaggar, ${ }^{6,7}$ Rabindra Tirouvanziam, ${ }^{4,5}$ and Krishnendu Roy ${ }^{1,2,3}$ \\ 'Wallace H. Coulter Department of Biomedical Engineering, Georgia Institute of Technology and Emory University, Atlanta, \\ Georgia, USA. ${ }^{2}$ Center for Immunoengineering, Georgia Institute of Technology, Atlanta, Georgia, USA. ${ }^{3}$ National Science \\ Foundation (NSF) Engineering Research Center for Cell Manufacturing Technologies, Atlanta, Georgia, USA. ${ }^{4}$ Department \\ of Pediatrics, Emory University School of Medicine, Atlanta, Georgia, USA. ${ }^{5}$ Center for CF \& Airways Disease Research, \\ Children's Healthcare of Atlanta, Atlanta, Georgia, USA. ${ }^{6}$ Department of Medicine, University of Alabama at Birmingham, \\ Birmingham, Alabama, USA. ${ }^{7}$ Birmingham VA Medical Center, Birmingham, Alabama, USA.
}

Pulmonary drug delivery presents a unique opportunity to target lower airway inflammation, which is often characterized by the massive recruitment of neutrophils from blood. However, specific therapies are lacking modulation of airway neutrophil function, and difficult challenges must be overcome to achieve therapeutic efficacy against pulmonary inflammation, notably drug hydrophobicity, mucociliary and macrophage-dependent clearance, and high extracellular protease burden. Here, we present a multistage, aerodynamically favorable delivery platform that uses extracellular proteolysis to its advantage to deliver nanoparticle-embedded hydrophobic drugs to neutrophils within the lower airways. Our design consists of a self-regulated nanoparticle-inmicrogel system, in which microgel activation is triggered by extracellular elastase (degranulated by inflammatory neutrophils), and nanoparticles are loaded with Nexinhib20, a potent neutrophil degranulation inhibitor. Successful in vivo delivery of Nexinhib20 to the airways and into neutrophils promoted resolution of the inflammatory response by dampening neutrophil recruitment and degranulation, proinflammatory cytokine production in both airway and systemic compartments, as well as the presence of neutrophil-derived pathological extracellular vesicles in the lung fluid. Our findings showcase a new platform that overcomes challenges in pulmonary drug delivery and allows customization to match the proteolytic footprint of given diseases.

Authorship note: JCM, OAF, and CM are co-first authors. $A G, R T$, and $K R$ are co-senior authors.

Conflict of interest: The authors have declared that no conflict of interest exists.

Copyright: (c) 2019, American Society for Clinical Investigation.

Submitted: July 2, 2019 Accepted: October 23, 2019 Published: December 5, 2019.

Reference information: /CI Insight. 2019;4(23):e131468. https://doi.org/10.1172/jci. insight.131468.

\section{Introduction}

Intractable lung inflammation in patients with acute respiratory distress syndrome (ARDS), chronic obstructive pulmonary disease (COPD), and cystic fibrosis (CF) is characterized by sustained and nonresolving polymorphonuclear neutrophil (PMN) recruitment to the airways (1-4). Subsequent extracellular release and accumulation of PMN-derived proteases, such as neutrophil elastase (NE) and matrix metalloproteinase-9 (MMP-9), lead to the saturation of the antiprotease shield and unopposed proteolytic action, resulting in structural lung damage and decline in lung function $(5,6)$. Repackaging of released NE into extracellular vesicles (EVs) also leads to a pathological conformation that is inherently resistant to the lung antiprotease shield and promotes emphysema (7). The presence of these proteases in the airway milieu is linked to progression of structural lung damage (8-10). Thus, targeting of airway PMN granule and EV release is of significant therapeutic importance, with potential for broad clinical impact (11). However, to date, the standard of care relies on the prescription of antiinflammatories delivered systemically or on inhaled corticosteroids, which do not target effectively the neutrophilic component of the inflammatory response.

Pulmonary drug delivery offers an alternative route for direct administration of therapeutics to airway PMNs, with the added potential to reduce the overall dose needed and reduce possible systemic toxicity. One challenge to the pulmonary delivery of nonencapsulated drugs is the highly proteolytic microenvironment in pathological areas, which can severely reduce their efficacy (12-14). The hydrophobic nature of 
A

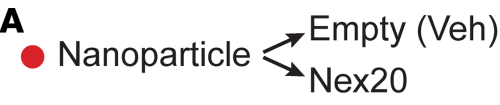<smiles>CCCCC(C)C</smiles>

HS-

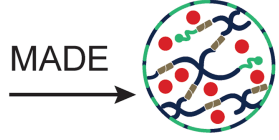

DL488-Mal

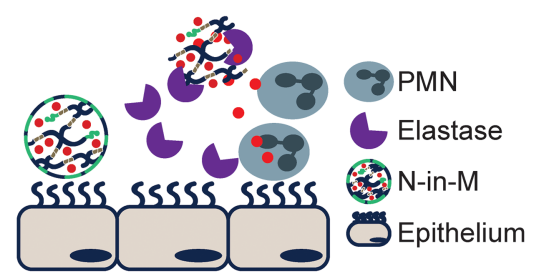

B
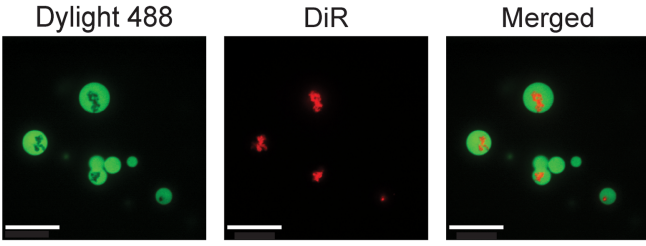

C

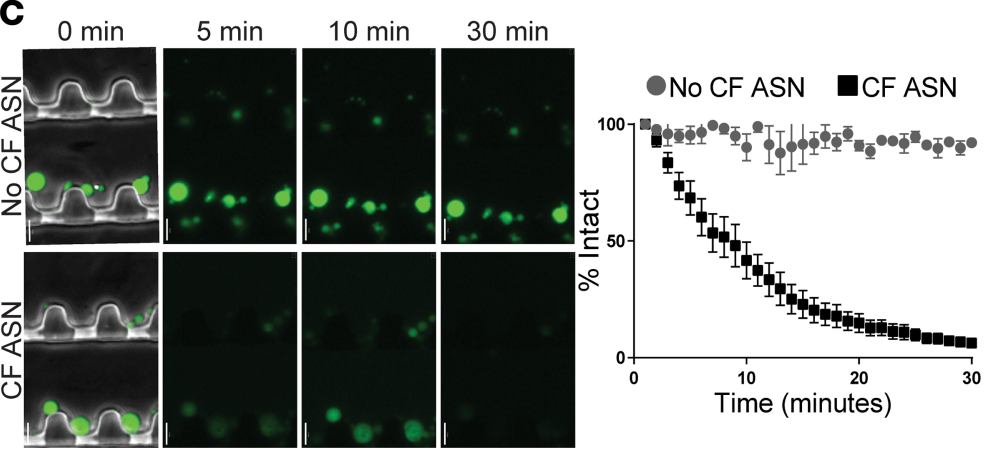

D

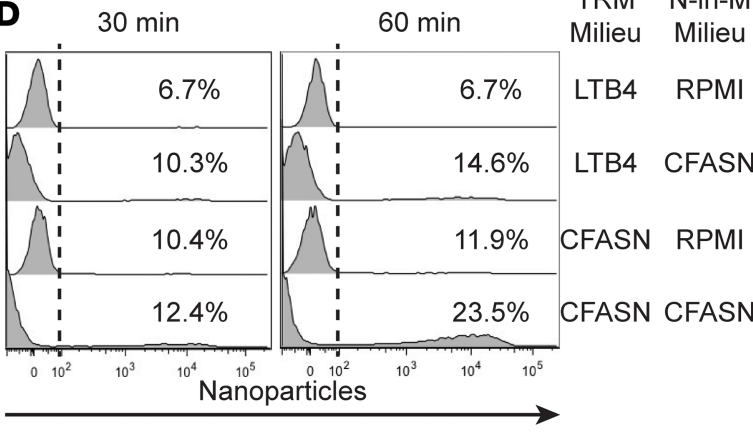

Figure 1. NE-responsive N-in-M degrades in cystic fibrosis airway supernatant, releasing fluorescent nanoparticles for endocytosis by PMNs. (A) Schematic of the $\mathrm{N}$-in-M system, demonstrating the structure of the $\mathrm{N}$-in-M system and showing the mechanism of nanoparticle release. The functional group sulfhydryl is both SH and HS; the bond is to the S. PEG, polyethylene glycol; MAL, maleimide; SH, sulfhydryl. (B) DiR-loaded poly(lactic-co-glycolic acid) (PLGA) nanoparticles encapsulated in DyLight 488-labeled elastase-responsive microgels; scale bar: $20 \mu \mathrm{m}$. (C) The N-in-M system degrades in the presence of high NE concentration (CF ASN) within 30 minutes; scale bar: $10 \mu \mathrm{m}$, mean \pm SEM; $n=3$ (no CF ASN), and $n=6$ (CF ASN). (D) PMNs transmigrated (TRM) to CF ASN readily show uptake of fluorescent nanoparticles encapsulated in NE-responsive microgels when the N-in-M is delivered to CF ASN.

some drugs, such as Nexinhib20, a potent PMN degranulation inhibitor (15), also hampers direct delivery. Embedding of drugs into particles could therefore improve pulmonary delivery. However, efficient particle-aided drug delivery imposes paradoxical constraints on particle size: those with a geometric diameter between 1 and $5 \mu \mathrm{m}$ are readily phagocytosed by lung macrophages, resulting in rapid clearance $(16,17)$, while only those smaller than $0.3 \mu \mathrm{m}$ enable effective intracellular delivery $(18,19)$.

To overcome these challenges, we fabricated a nanoparticle-in-microgel $(\mathrm{N}$-in-M) using a modified Michael addition during water-in-oil-emulsion method (20). The swellable microgel part of this multistage formulation provides the appropriate aerodynamic size for deep lung deposition in the polymer's relaxed state and appropriate geometric size to avoid alveolar macrophage clearance in the swollen state. Meanwhile, a cross-linked NE-degradable peptide incorporated into the microgel backbone allows for the rapid degradation of the microgel in the presence of NE to release Nexinhib20-loaded nanoparticles. We demonstrate that these nanoparticles allowed for efficient delivery of Nexinhib20 to the airway and into PMNs, leading to substantial blockade of systemic and lung inflammation.

\section{Results}

$N$-in-M degrades in NE-rich patient airway fluid, resulting in uptake of nanoparticles by PMNs. Targeting PMNs in vivo, particularly in highly proteolytic microenvironments, is limited by our ability to effectively deliver drugs to these cells. The multistage $\mathrm{N}$-in-M particle delivery system we designed allows for the controlled release of nanoparticles in regions of the lung with high concentration of proteolytic enzymes, specifically NE (Figure 1, A and B). The polymers used for both the nano and micro portions of the formulation were chosen because of their positive regulatory history and biodegradable properties. The swollen microgel geometric diameter is $3.9 \mu \mathrm{m}$ (mean, $8 \mu \mathrm{m}$ range), while encapsulated nanoparticles are under $200 \mathrm{~nm}$ (Supplemental Table 1 and Supplemental Figure 1; supplemental material available online with this article; https://doi.org/10.1172/jci.insight.131468DS1). Detailed physiochemical characterization of such microgels encapsulating representative polystyrene nanoparticles, and their biodistribution in naive mice, are reported elsewhere (21). To test the degradation of the multistage system cross-linked with a pulmonary disease-relevant NE-cleavable peptide, we imaged the $\mathrm{N}$-in-M during exposure at $37^{\circ} \mathrm{C}$ to pathologically high levels of NE in CF airway supernatant (CF ASN). Within 30 minutes of exposure to NE-rich CF 
A $\quad 1 \mathrm{~h}$

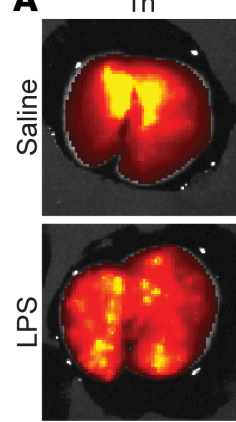

C

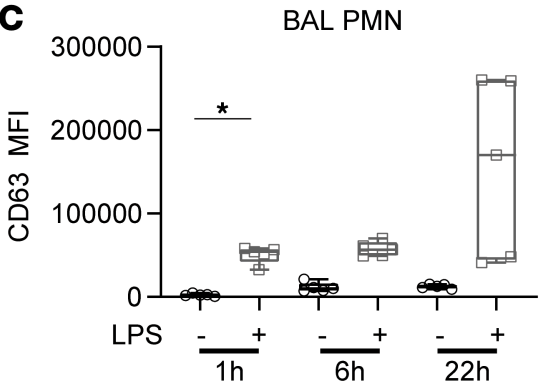
$22 \mathrm{~h}$
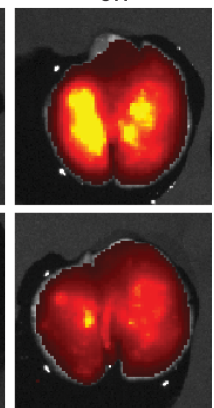
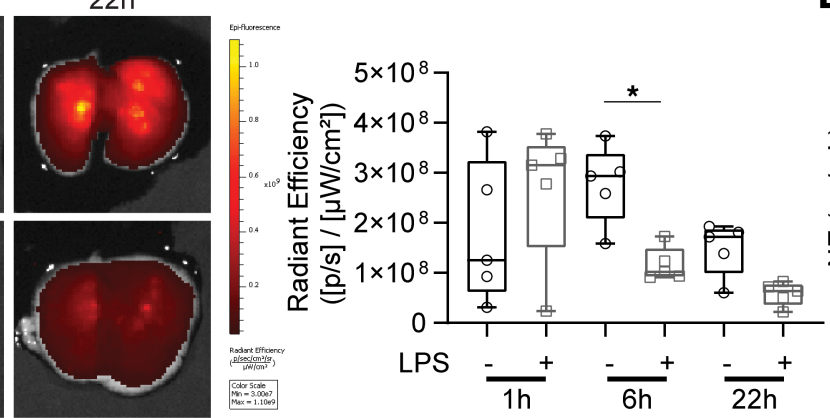

B

D

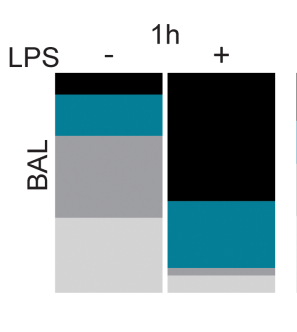

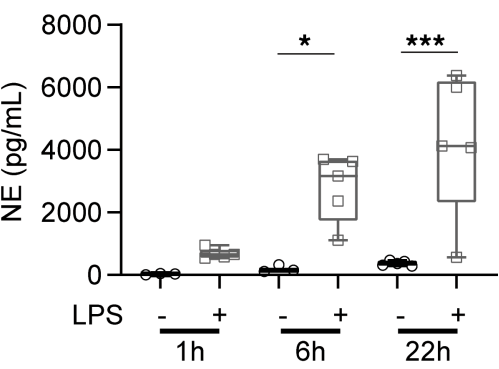

Figure 2. $\mathbf{N}$-in-M in an acute neutrophilic inflammation model. (A) Representative in vitro imaging system (IVIS) images of excised mouse lungs with corresponding measures of radiant efficiency for 1, 6, and 22 hours for saline- and LPS-treated mice. (B) Increase in BAL NE levels for the LPS-treated mice. (C) Increased CD63 expression on BAL PMNs. (D) Percentage of BAL PMNs positive for nanoparticle only, N-in-M, and microgel only or negative for both. Box plots show median, interquartile range (IQR), and min to max. Statistical tests: Shapiro-Wilk followed by 2-way ANOVA (A) and Kruskal-Wallis (B and C). ${ }^{*} P<0.05$, and ${ }^{* *} P<0.001$ within time points indicated. $n=5$ per group.

ASN, $95 \%$ of the microgels had degraded (Figure 1C), which shows that our N-in-M system should quickly release nanoparticles if delivered to protease-rich airways.

Next, we assessed delivery of our N-in-M system to PMNs in vitro. This was accomplished in a PMN transmigration model (22), which uses the relevant biological milieu, i.e., CF ASN, as the apical fluid and has been shown previously to recapitulate the in vivo phenotype of PMNs found in CF patients' airways (23). This model was also used to recapitulate PMN adaptation occurring in pediatric ARDS (24). Here, we measured uptake by airway PMNs of 60-nm fluorescent carboxylated polystyrene nanoparticles encapsulated within the NE-degradable microgels and observed that the degradation of the microgels in the presence of NE-rich CF ASN increased the percentage of PMNs positive for the nanoparticle by $5 \%-10 \%$ at 60 minutes, regardless of the transmigration milieu (chemoattractant control consisting of leukotriene B4 [LTB4] or CF ASN). When both PMNs' transmigration and the N-in-M exposure occurred in the context of CF ASN, uptake of fluorescent nanoparticles by PMNs was increased (Figure 1D). Together, these data indicate that for efficient uptake of nanoparticles by airway PMNs, the NE-responsive microgel must degrade first. The additive effect between the acquired PMN phenotype, imprinted by the transmigration condition, and the incubation milieu with the $\mathrm{N}$-in-M system suggests that targeting of airway PMNs by the N-in-M system would be more efficient in pathological settings (CF ASN) rather than during the normal course of inflammation (LTB4).

$N$-in-M administered to an acute LPS-induced lung injury mouse model degrades, delivering representative fluorescent nanoparticles to airway PMNs. Next, we tested the efficacy of the N-in-M multistage particle system in an in vivo inflammation model characterized by high levels of extracellular NE (25). The $\mathrm{N}$-in-M system consisting of microgels labeled with the near IR dye DyLight 650 loaded with representative blue (350/440) FluoSpheres nanoparticles was delivered intratracheally to mice treated with saline or LPS. The radiant efficiency in the excised lungs, corresponding to the fluorescence intensity of the microgel dye at 1, 6, and 22 hours after N-in-M delivery ( 3,8 , and 24 hours after LPS), showed a significant reduction at 6 hours after $\mathrm{N}$-in-M delivery (Figure 2A), revealing a faster microgel degradation in mice treated with LPS than in saline-treated controls.

Concurrent with microgel degradation, we observed a reduction over time of blood PMN activation and PMN recruitment to the airways and subsequent release of NE-rich granules (reflected by surface CD63) and amount of soluble NE in the bronchoalveolar lavage (BAL) (Figure 2, B and C). 
A

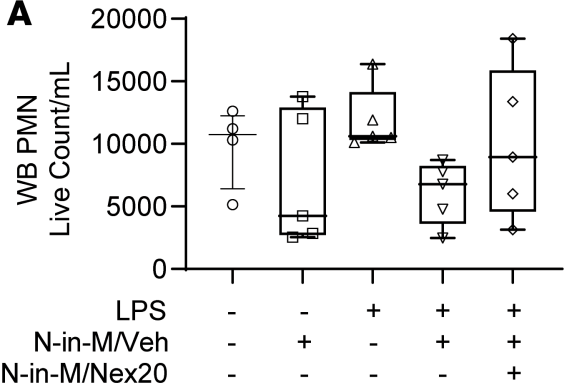

$\mathrm{N}$-in-M/Nex20

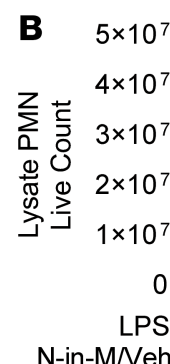

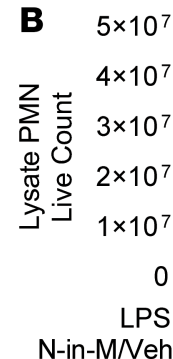
$\mathrm{N}-\mathrm{in}-\mathrm{M} / \mathrm{Nex} 20$
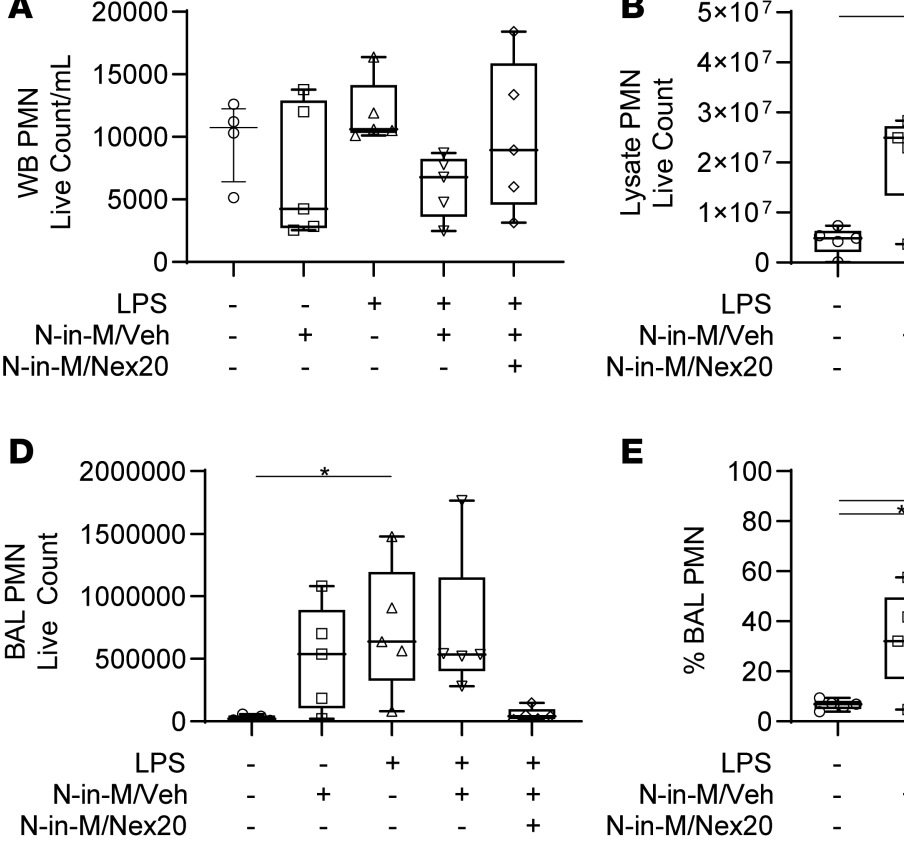

E

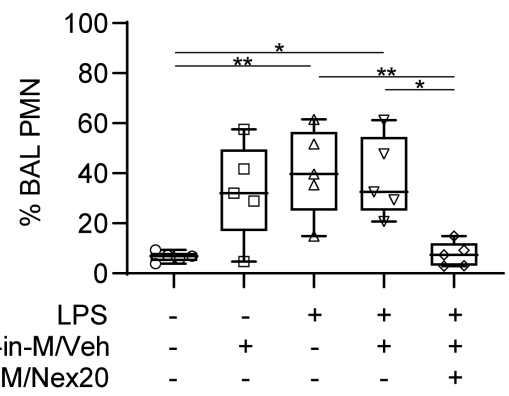

C

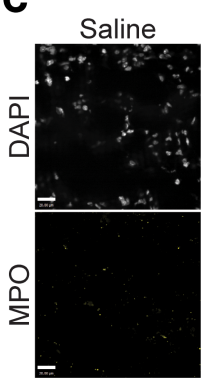

LPS

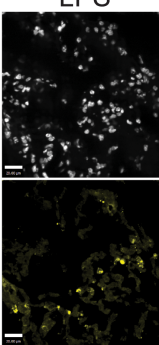

$\mathrm{N}$-in-M Nex20

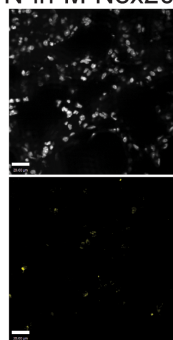

$\mathbf{F}$

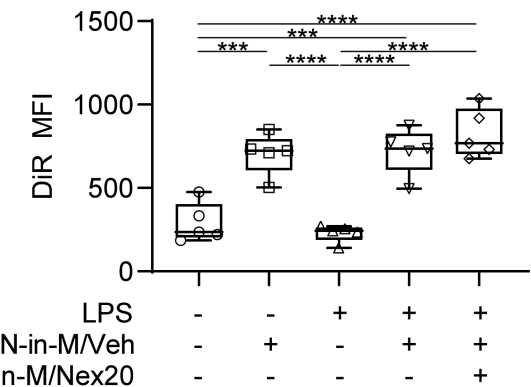

Figure 3. $\mathbf{N}$-in-M delivery of Nexinhib20 attenuates neutrophilic inflammation. (A) Blood showed no detectable signs of neutrophilia or neutropenia. Lung lysate absolute PMN count (B) and presence of myeloperoxidase-positive PMNs in the tissue (C) were decreased upon treatment with Nexinhib20. In the BAL, expected increases following LPS treatment of PMN absolute count (D) and percentage (E) were abrogated in the Nexinhib20 treatment group, concomitant with PMN uptake of the $\mathrm{N}$-in-M system $(\mathbf{F})$, as measured by DiR nanoparticle fluorescence. Saline was administered to the control group. Box plots show median, IQR, and min to max. Statistical tests: Shapiro-Wilk followed by 1-way ANOVA (E and F) and Kruskal-Wallis (A, B, and D), with ${ }^{*} P<0.05,{ }^{* *} P<0.01,{ }^{* *} P<0.001$, and ${ }^{* * *} P<0.0001$ between groups indicated. $n=5$ per group.

Thus, the short-term clearance of microgels during LPS treatment is preceded by and dependent upon the degradation of the NE-sensitive peptides cross-linking it. Notably, the population of BAL PMNs double positive for the microgel and nanoparticles showed increased surface CD63 expression, suggesting that fusion to the plasma membrane of NE-rich granules and release of their content in the extracellular milieu is concomitant with increased uptake of soluble material, as shown before (22). Last, analysis of the distribution of microgel and nanoparticle uptake showed that airway PMNs at 1 and 6 hours after delivery were primarily positive for nanoparticles alone, suggesting that microgels were degraded, allowing for nanoparticle endocytosis (Figure 2D). These findings showcase the efficacy of the N-in-M system for nanoparticle delivery to airway PMNs in a protease-rich environment in vivo.

$N$-in-M delivery of Nexinhib20 attenuates neutrophilic inflammation in vivo. To determine whether the $\mathrm{N}$-in-M system could not only effectively deliver nanoparticles to PMNs but also modulate PMN-driven inflammation in vivo, Nexinhib20- and DiR-carrying PLGA nanoparticles were loaded into the NE-degradable microgels (N-in-M/Nex), with DiR, a near IR fluorescent dye serving as a tracer for nanoparticle uptake. To assess whether the N-in-M system with PLGA influenced the immune response, additional control groups for both saline- and LPS-treated mice were treated with vehicle DiR-only PLGA nanoparticles inside the NE-degradable microgels (N-in-M/Veh).

Although there was no detectable sign of neutrophilia or neutropenia in blood (Figure 3A), N-in-M/ Nex reduced the percentage (Figure 3, B-E, and Supplemental Figure 2) and mean total number of PMNs in the lung lumen and lung parenchyma compared with the LPS-treated control group, by 13- and 4.3-fold, respectively. These results, in addition to the detection of DiR dye in airway PMNs (Figure 3F), suggests successful delivery of the drug to PMNs by the N-in-M system. Next, we investigated whether the reduction in PMN in the airways was reflective of a decrease in inflammatory poise. Analysis of critical BAL and plasma cytokines revealed a significant decrease in chemoattractants implicated in PMN and macrophage recruitment, KC/GRO and MIP-2 (Supplemental Figure 3, A-D), and the proinflammatory cytokines TNF- $\alpha$, IL-6, and IL-1 $\beta$ (Supplemental Figure 3, E-J). Extensive mediator profiling in plasma and BAL confirmed the reduction in inflammatory signaling induced by treatment with $\mathrm{N}-\mathrm{in}-\mathrm{M} / \mathrm{Nex}$, both locally and systemically (Supplemental Figure 4). 
A

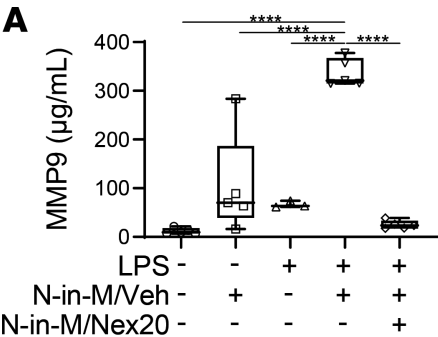

C

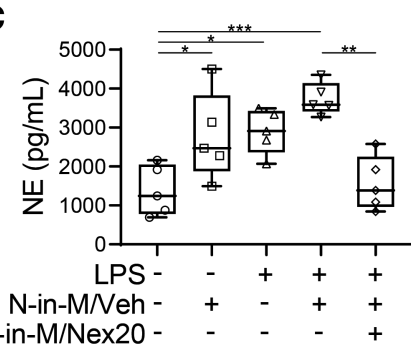

B

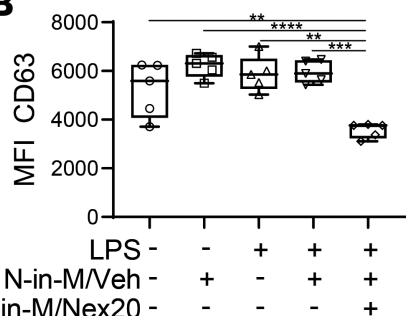

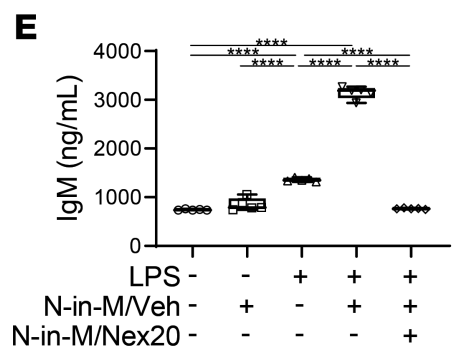

D

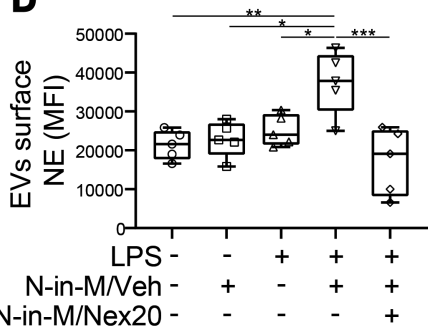
$\mathrm{N}$-in-M/Nex20 -

Figure 4. Delivery of Nexinhib20 reduces PMN degranulation in the lung. (A) Treatment with Nexinhib20 reduced active release by BAL neutrophils of soluble MMP-9. Primary granule release, measured by surface CD63 (B), soluble NE (C), and EV-bound NE measured in the BAL (D), also showed that significant increases by LPS and control PLCA $\mathrm{N}$-in-M were abrogated by Nexinhib20 delivery. (E) Reduction of the overall inflammatory response upon delivery of Nexinhib20 led to reduced capillary leakage measured by IgM levels in the BAL fluid. Saline was administered to the control group. Box plots show median, IQR, and min to max. Statistical tests: Shapiro-Wilk followed by 1-way ANOVA. ${ }^{*} P<0.05$, ${ }^{* *} P<0.01$, ${ }^{* *} P<0.001$, and ${ }^{* * *} P<0.0001$ between groups indicated. $n=5$ per group.

To determine whether $\mathrm{N}$-in-M/Nex could influence not only PMN numbers, but also their phenotype and function, active granule release was assessed by ELISA and flow cytometry. Despite similar PMN recruitment in LPS-alone controls, in N-in-M/Veh-alone controls, and in N-in-M/Veh LPS-treated mice, MMP-9 levels in the latter showed a 28-fold increase compared with the saline treatment group and a 5-fold increase compared with the LPS-alone group (Figure 4A). Delivery of Nexinhib20 abrogated this response in N-in-M/Nex mice, supporting the effectiveness of this potentially novel delivery system at not only counteracting LPS-induced inflammation but also dampening potential inflammatory responses induced by the delivery of the $\mathrm{N}$-in-M system itself, likely caused by the detection of foreign particles or by delivery-associated tissue trauma. Most strikingly, concomitant with increased nanoparticle uptake by PMNs, surface CD63 expression on BAL PMNs (Figure 4B), reflective of NE release, was significantly lower for the Nexinhib20 formulation than all the other treatment groups. Likewise, N-in-M/Nex treatment lowered the amount of extracellular NE (Figure 4C) and presence of NE-rich EVs (Figure 4D), a pathological NE conformation we previously showed to be resistant to the lung antiprotease shield and thereby inducing emphysema (7). Last, the successful reduction of damaging enzymes in the extracellular compartment, with the dampening of the inflammatory response upon delivery of the $\mathrm{N}$-in-M/Nex system, was reflected in the health of the lung tissue and reduction in inflammation-induced capillary leak, as reflected by IgM levels in the BAL fluid (Figure 4E). 


\section{Discussion}

Together, our findings establish a potentially novel and specific delivery method for hydrophobic drug administration to airway PMNs. The protease-sensitive design of the N-in-M system not only allows the microgel to provide the needed aerodynamic size for deposition and geometric size to avoid rapid clearance ( $<1$ hour), but it also exploits the high proteolytic microenvironment for controlled release of drug-loaded nanoparticles. Furthermore, encapsulation of the drug in the nanoparticle shields it and preserves its therapeutic potential. Although the system presented here was designed to be effective in the presence of NE, it presents ample opportunities for customization because the protease-sensitive peptides embedded in the microgel can be tailored to a plethora of cleavage sites specific to other enzymes. Moreover, the N-in-M system can be made to accommodate virtually any drug. Here, we chose to include Nexinhib20 because its main target is actually exocytosis and downstream NE release. Therefore, we accomplished efficient NE-dependent delivery of a drug targeting the machinery responsible for the release of $\mathrm{NE}$ itself, which led to the resolution of inflammation, eventually preventing further degradation of new microgels (i.e., self-limiting antiinflammatory therapy, as illustrated in the graphical abstract).

In addition to mucociliary clearance, another major challenge for inhaled drug delivery is that, because of progressive airway damage and heterogeneous worsening of pulmonary ventilation and perfusion matching, it is difficult to have adequate deposition of the drug into diseased areas of the lung (26). This remains a major problem for therapeutic development and has limited the effectiveness and safety of inhaled medications in chronic lung disease. Although we anticipate some limitation in delivery because of disease pathophysiology, our disease-targeted $\mathrm{N}$-in-M system takes advantage of protease-specific drug release, which provides the ability to direct the active pharmaceutical ingredient primarily to areas of active inflammation and remodeling.

Another critical point in inhaled therapeutic development is the ability to tune the clinical response to provide benefit without incurring additional pathology or side effects (27). When using antiinflammatory agents, it is imperative to recognize the often narrow therapeutic windows, which may reduce healthy host responses, such as release of antimicrobial enzymes and neutrophil extracellular traps, while targeting progressive tissue damage. A benefit of the protease-responsive $\mathrm{N}$-in-M system is that the release of the drug is self-limited by the loss of excessive protease activity. This approach allows for localized and measured delivery of antiinflammatory drugs to the diseased tissue, allowing for enhanced safety compared with nontargeted delivery of antiinflammatory agents to the whole lung.

Last, these results highlight the importance of appropriate drug selection when using foreign particles for therapeutic delivery. In this study, the addition of the empty $\mathrm{N}$-in-M demonstrated some increase in inflammatory metrics (i.e., cell count, permeability), which was further exacerbated in the inflamed lung (LPS treatment). This is a key challenge in pulmonary delivery to diseased lungs. However, the delivery of the appropriate drug (Nexinhib20) in the N-in-M system completely reshaped the inflammatory landscape to the levels of the saline-treated group, confirming the effectiveness and safety of this system.

In conclusion, our findings establish the potential of $\mathrm{N}$-in-M delivery systems to effectively target inflammation in pulmonary disorders characterized by unrelenting PMN influx and activation. Similar $\mathrm{N}$-in-M systems may provide a relevant mechanism to strategically deliver a variety of therapeutics not only to the lung but also to other organs with high proteolytic burden (e.g., inflamed gut, joints, and tumors).

\section{Methods}

Nanoparticle and $N$-in-M fabrication. Therapeutics and/or dye were encapsulated in nanoparticles during fabrication using a single emulsion solvent evaporation method. Then, 100-nm Blue FluoSpheres (Thermo Fisher Scientific) or lyophilized PLGA nanoparticles were resuspended and encapsulated in 20\% w/v elastase-responsive microgels using a Michael addition during water-in-oil emulsion. See details in Supplemental Methods.

In vitro transmigration experiments. Blood PMNs were isolated using Polymorphprep (Alere Technologies) as per the manufacturer's protocol, washed, and then loaded on the transmigration chamber; transmigration and following incubations were performed using $100 \mathrm{nM}$ of LTB4 (MilliporeSigma) in RPMI or CF ASN, prepared as previously published (22). The uptake of fluorescent microgels was then assessed 10 hours after transmigration by incubating PMNs recruited apically with microgels at a 1:10 ratio (PMN/ microgel) in CF ASN or RPMI for 30 and 60 minutes and quantified using flow cytometry.

Flow cytometry. Cells from in vitro and in vivo assays were analyzed by multiparametric flow cytometry, as previously detailed (28), and gated as shown in Supplemental Figure 5. See Supplemental Methods for details and Supplemental Table 2 for antibodies' characteristics. 
EV analysis. EVs were isolated from the BAL of mice using bead-based pull-down with annexin $\mathrm{V}$, as per the manufacturer's protocol (SBI). Isolated EVs were stained with the EV marker ExoFITC (SBI) and anti-mouse NE (R\&D Systems). EVs were analyzed by flow cytometry on a Cytoflex S (Beckman Coulter).

Fluid assays. NE and MMP-9 concentrations were measured by ELISA in mouse BAL fluid per the manufacturer's protocol (R\&D Systems). Inflammatory mediators in plasma and BAL fluid were measured using a multiplex chemoluminescent platform per the manufacturer's protocol (MesoScale Diagnostic, V-PLEX mouse cytokines, 19 analytes). BAL fluid IgM concentration was measured by ELISA per the manufacturer's protocol (Immunology Consultants Laboratory).

Microscopy. N-in-Ms were imaged on a PerkinElmer UltraVIEW VoX spinning disk confocal microscope with a Hamamatsu C9100-23b back-thinned EM-CCD camera and Nikon 100× NA-1.45 oil objective; lung sections were imaged with the Nikon 40× NA-1.3 oil objective. Microgel size was measured in the relaxed state using Volocity (PerkinElmer Inc.) and in the swollen state using MATLAB "imfindcircles"; this does cap the lower size limit measured. Degradation was measured by kinetic imaging every minute using a BioTek Lionheart at $37^{\circ} \mathrm{C}$ using cell traps (Lu lab at the Georgia Institute of Technology) to hold the microgels in place (29).

Animal studies. Mice were treated with either saline or $100 \mu \mathrm{g}$ of LPS. Then for the fluorescent uptake study, the N-in-M formulation was delivered 2 hours later and for the Nexinhib20 study 1 hour after injection. The time for N-in-M injection was shortened because at 2 hours after LPS mice had significant difficulty in aspirating the particle formulation. Mice used for analysis with an IVIS were given a low-alfalfa feed diet to reduce fluorescence background. Excised lobes were imaged on an IVIS and analyzed by LivingImage software (Xenogen). Further analysis of excised lobes by immunofluorescent staining was performed per established protocols, as detailed in the Supplemental Methods.

Statistics. Data were analyzed in Prism (GraphPad). The Shapiro-Wilk test was used to assess normality $(\alpha=0.05)$. For parametric data, we used 2-sided 1-way ANOVA and 2-way ANOVA $(\alpha=0.05)$ with post hoc Tukey's test with adjusted $P$ value for multiple comparisons. For nonparametric data, Kruskal-Wallis ( $\alpha=0.05$ ) with Dunn's multiple-comparisons test with adjusted $P$ value was used. The $P$ value results of the statistical tests were compared with the $\alpha$ level. If the $P$ value was less than the $\alpha$ level, then the null hypothesis was rejected, and the result was statistically significant.

Study approval. Blood was collected from healthy donors after informed consent was obtained according to an approved Emory University Institutional Review Board protocol. Animal studies were performed according to protocols approved by the IACUC at the University of Alabama Birmingham. Five-week-old female C57BL/6 littermates (Jackson Laboratories) were maintained in a pathogen-free facility and given sterile water and food ad libitum.

\section{Author contributions}

JCM, OAF, CM, AG, RT, and KR conceived the studies. JCM, OAF, CM, and XX conducted primary experiments and performed data analyses. DAFR, LV, and JL provided critical support to experiments. JCM, OAF, CM, AG, RT, and KR wrote the manuscript.

\section{Acknowledgments}

The authors thank the Lu lab at the Georgia Institute of Technology for the use of cell traps and the Parker $\mathrm{H}$. Petit Institute for Bioengineering and Bioscience at the Georgia Institute of Technology and the Emory University Pediatrics Flow Cytometry Core for the use of their facilities.

This work was supported by the Center for Cystic Fibrosis Airways Disease Research pilot grant and Children's Healthcare of Atlanta (AG, RT, KR), Robert A. Milton Chair funds (KR), funds from the Georgia Tech Foundation (KR), NSF DMR 1417137 (KR), NIH/National Institute of General Medical Sciences-sponsored Cell and Tissue Engineering Biotechnology Training Program T32GM008433 (JCM), NIH R01 HL102371 (AG), NIH R01 HL126603 (RT), NSF Graduate Research Fellowship DGE-1650044 (JCM), and CF@LANTA Research Development Program Fellowship (CM) as funded by the US Cystic Fibrosis Foundation (MCCART15R0).

Address correspondence to: Rabindra Tirouvanziam, Emory Children's Center, 2015 Uppergate Drive Northeast, Room 344, Atlanta, Georgia 30322, USA. Phone: 404.712.7684; Email: tirouvanziam@emory.edu. Or to: Krishnendu Roy, Experimental Biosystems Building, 950 Atlantic Drive, Room 3018, Atlanta, Georgia 30332, USA. Phone: 404.385.6166; Email: krish.roy@gatech.edu. 
1. Grommes J, Soehnlein O. Contribution of neutrophils to acute lung injury. Mol Med. 2011;17(3-4):293-307.

2. Hoenderdos K, Condliffe A. The neutrophil in chronic obstructive pulmonary disease. Am J Respir Cell Mol Biol. 2013;48(5):531-539.

3. Margaroli C, Tirouvanziam R. Neutrophil plasticity enables the development of pathological microenvironments: implications for cystic fibrosis airway disease. Mol Cell Pediatr. 2016;3(1):38.

4. De Rose V, Burgel PR, Gaggar A, Greene C. Airway inflammatory/immune responses in COPD and cystic fibrosis. Mediators Inflamm. 2018;2018:7280747.

5. Gaggar A, Hector A, Bratcher PE, Mall MA, Griese M, Hartl D. The role of matrix metalloproteinases in cystic fibrosis lung disease. Eur Respir J. 2011;38(3):721-727.

6. Wagner CJ, Schultz C, Mall MA. Neutrophil elastase and matrix metalloproteinase 12 in cystic fibrosis lung disease. Mol Cell Pediatr. 2016;3(1):25.

7. Genschmer KR, et al. Activated PMN exosomes: pathogenic entities causing matrix destruction and disease in the lung. Cell. 2019;176(1-2):113-126.e15.

8. Griese M, Kappler M, Gaggar A, Hartl D. Inhibition of airway proteases in cystic fibrosis lung disease. Eur Respir J. 2008;32(3):783-795.

9. Guyot N, et al. Unopposed cathepsin G, neutrophil elastase, and proteinase 3 cause severe lung damage and emphysema. Am J Pathol. 2014;184(8):2197-2210.

10. Kruger P, et al. Neutrophils: between host defence, immune modulation, and tissue injury. PLoS Pathog. 2015;11(3):e1004651.

11. Gernez Y, Tirouvanziam R, Chanez P. Neutrophils in chronic inflammatory airway diseases: can we target them and how? Eur Respir J. 2010;35(3):467-469.

12. Brigden G, du Cros P, Wong S. Barriers to new drug development in respiratory disease. Eur Respir J. 2016;47(1):356-357.

13. Ibrahim BM, Tsifansky MD, Yang Y, Yeo Y. Challenges and advances in the development of inhalable drug formulations for cystic fibrosis lung disease. Expert Opin Drug Deliv. 2011;8(4):451-466.

14. Labiris NR, Dolovich MB. Pulmonary drug delivery. Part I: physiological factors affecting therapeutic effectiveness of aerosolized medications. Br J Clin Pharmacol. 2003;56(6):588-599.

15. Johnson JL, et al. Identification of neutrophil exocytosis inhibitors (nexinhibs), small molecule inhibitors of neutrophil exocytosis and inflammation: druggability of the small GTPase Rab27a. J Biol Chem. 2016;291(50):25965-25982.

16. Edwards DA, et al. Large porous particles for pulmonary drug delivery. Science. 1997;276(5320):1868-1871.

17. Patel B, Gupta N, Ahsan F. Particle engineering to enhance or lessen particle uptake by alveolar macrophages and to influence the therapeutic outcome. Eur J Pharm Biopharm. 2015;89:163-174.

18. Schneider CS, et al. Nanoparticles that do not adhere to mucus provide uniform and long-lasting drug delivery to airways following inhalation. Sci Adv. 2017;3(4):e1601556.

19. Zhang S, Gao H, Bao G. Physical principles of nanoparticle cellular endocytosis. ACS Nano. 2015;9(9):8655-8671.

20. Wanakule P, Liu GW, Fleury AT, Roy K. Nano-inside-micro: disease-responsive microgels with encapsulated nanoparticles for intracellular drug delivery to the deep lung. J Control Release. 2012;162(2):429-437.

21. Mejías JC, Roy K. In-vitro and in-vivo characterization of a multi-stage enzyme-responsive nanoparticle-in-microgel pulmonary drug delivery system. J Control Release. In press.

22. Forrest OA, et al. Frontline Science: pathological conditioning of human neutrophils recruited to the airway milieu in cystic fibrosis. J Leukoc Biol. 2018;104(4):665-675.

23. Mitchell TC. A GRIM fate for human neutrophils in airway disease. J Leukoc Biol. 2018;104(4):657-659.

24. Grunwell JR, et al. Neutrophil dysfunction in the airways of children with acute respiratory failure due to lower respiratory tract viral and bacterial coinfections. Sci Rep. 2019;9(1):2874.

25. Corteling R, Wyss D, Trifilieff A. In vivo models of lung neutrophil activation. Comparison of mice and hamsters. $B M C$ Pharmacol. 2002;2:1.

26. Bos AC, et al. Patient-specific modeling of regional antibiotic concentration levels in airways of patients with cystic fibrosis: are we dosing high enough? PLoS ONE. 2015;10(3):e0118454.

27. Barnes PJ. New anti-inflammatory targets for chronic obstructive pulmonary disease. Nat Rev Drug Discov. 2013;12(7):543-559.

28. Tirouvanziam R, Diaz D, Gernez Y, Laval J, Crubezy M, Makam M. An integrative approach for immune monitoring of human health and disease by advanced flow cytometry methods. In: Tuchin V, ed. Advanced Optical Flow Cytometry. Weinheim, Germany: Wiley-VCH Verlag; 2011:333-362.

29. Chung K, Rivet CA, Kemp ML, Lu H. Imaging single-cell signaling dynamics with a deterministic high-density single-cell trap array. Anal Chem. 2011;83(18):7044-7052. 